\title{
Microbial Ecology of the Murine Gut Associated with the Development of Dextran Sodium Sulfate-Induced Colitis
}

\author{
Nabeetha A. Nagalingam, MPhil, ${ }^{*}$ John Y. Kao, MD, ${ }^{+}$and Vincent B. Young, MD, PhD ${ }^{\neq, \S}$
}

\begin{abstract}
Background: Dextran sodium sulfate (DSS) is used to induce murine colitis. Although the exact mechanism by which DSS administration causes disease is unknown, evidence suggests that the resident bacteria play a role in the development of murine DSS colitis, analogous to their role in human inflammatory bowel diseases.
\end{abstract}

Methods: C57BL/6 mice received 5\% DSS in the drinking water and were euthanized 3 days and 14 days after the initiation of DSS treatment. Culture-independent methods were used to follow changes in the community structure of the gut's microbiota following DSS treatment. Histologic evidence of disease and changes in host gene expression were assessed.

Results: Histologic colitis was minimal in DSS-treated animals at 3 days, but severe after 14 days. Analysis of 16S rRNA-encoding gene clone libraries demonstrated that the microbial communities in the ceca of DSS-treated mice were distinct from those in control mice. The microbiota in the cecum of DSS-treated animals was characterized by an overall decrease in microbial richness, an increase in members of the phylum Verrucomicrobia, and decrease in Tenericutes. Changes in the host's inflammatory response and microbial communities occurred before the histologic appearance of severe disease in the colon, but were seen concurrently in the cecum.

Conclusions: DSS administration is associated with reproducible changes in the gut microbial diversity of mice. Microbial and immunological changes appeared before the development of severe inflammation in the colon. This indicates that these changes in microbial community may play role in the potentiation of the abnormal inflammatory response seen in DSS-treated animals.

(Inflamm Bowel Dis 2011;17:917-926)

Additional supporting information may be found in the online version of this article.

Received for publication July 22, 2010; Accepted July 26, 2010.

From the *Michigan State University, Department of Microbiology and Molecular Genetics, 'University of Michigan Medical School, Department of Internal Medicine/Gastroenterology Division, ${ }^{+}$University of Michigan Medical School, Department of Internal Medicine/Infectious Diseases Division, ${ }^{\S}$ University of Michigan, Department of Microbiology and Immunology, Ann Arbor, Michigan.

Supported by the Center for Microbial Pathogenesis, Michigan State University; National Institutes of Health (NIH) grant DK070875 (to V.B.Y.); Crohn's and Colitis Foundation of America Senior Investigator Award (to V.B.Y.).

Reprints: V.B. Young, 4618D Med. Sci II SPC 5623, 1150 W. Medical Center Dr., Ann Arbor, MI 48109-5623 (e-mail: youngvi@umich.edu).

Copyright (c) 2010 Crohn's \& Colitis Foundation of America, Inc.

DOI 10.1002/ibd.21462

Published online 8 November 2010 in Wiley Online Library (wileyonlinelibrary.com).
Key Words: microbiota, DSS, 16S rRNA-encoding gene, ecology, colitis

$\mathrm{M}$ icrobes are essential to the health and well-being of their hosts. ${ }^{1,2}$ However, the presence of bacteria alone is not enough to impart these benefits; rather, the composition and relative abundance of specific microbes have an important role in maintaining health. ${ }^{3}$ Alterations of the community structure of the indigenous microbiota have also been implicated in the development of disease. One such condition in which the resident bacteria are thought to play a critical role in pathogenesis is inflammatory bowel disease (IBD) ${ }^{3,4}$ Dysbiosis, defects in immunoregulation, and defects in the barrier function are all thought to contribute to the onset of disease..$^{5-7}$ Evidence of a key role for the microbiota in pathogenesis is provided by studies that demonstrate that antibiotics can reduce or prevent inflammation both in patients and in murine models of disease. ${ }^{8,9}$ In IL10 $0^{-1-}$ mice, which develop colitis after infection with certain bacteria or in the setting of certain housing conditions, treatment with antibiotics is associated with alterations in the microbial gut community ${ }^{10}$ and the prevention or amelioration of disease. ${ }^{11}$

Of the multiple murine models of IBD, one commonly used system involves the administration of dextran sodium sulfate (DSS), which induces disease very similar to human ulcerative colitis. ${ }^{12}$ Antibiotic administration has been shown to ameliorate DSS-induced colitis ${ }^{13}$ and cathelicidin, an antimicrobial peptide, was also found to have protective effects in this model of colitis, ${ }^{14}$ indicating that the microbiota plays a role in this disease model system.

Multiple hypotheses have been postulated for the mechanism by which DSS triggers gut mucosal inflammation but the exact pathogenesis remains unclear. ${ }^{14,15}$ Given that human IBD is associated with an altered microbial diversity $^{5,16}$ and evidence for a role of the microbiota in DSS-induced colitis, ${ }^{14,17}$ we hypothesize that this model is also associated with an altered diversity of the intestinal microbiota. To test whether DSS treatment can alter the microbial community diversity we employed molecular techniques targeting the 16S rRNA-encoding gene to follow the community structure of the gut bacteria in animals receiving DSS. We correlated changes in the community structure with development of disease and host responses. 


\section{MATERIALS AND METHODS}

Animals

C57BL/6 mice from a breeding colony initially established with breeding stock from Jackson Laboratories (Bar Harbor, ME) were used for experiments. The animal studies were conducted at Michigan State University and were approved by the Michigan State University Ethics Committee. Mice were housed with autoclaved bedding, given sterile food and water ad libitum, and exposed to 12:12-hour light:dark cycles. The mice, between 12 to 16 weeks old, were assigned to cages according to gender. DSS $(36,000$ 50,000 MW, ICN Biochemicals, Irvine, CA) was administered as a $5 \%$ solution in the drinking water. ${ }^{8}$

Three groups of mice were used in the experiment: 10 control mice that were maintained on sterile drinking water; 10 mice that were placed on 5\% DSS in sterile drinking water for 3 days before being euthanized; and 11 mice that were given $5 \%$ DSS for 14 days before euthanasia. ${ }^{18}$

\section{Necropsy and Histology}

The mice were euthanized by $\mathrm{CO}_{2}$ asphyxiation and the cecal tissue harvested as described previously. ${ }^{19}$ The tissues were gently washed with $1 \times$ phosphate-buffered saline (PBS) to remove the fecal contents, cut into sections, and snap-frozen in liquid nitrogen. One of these sections was used for terminal restriction fragment length polymorphism (T-RFLP) analyses and clone library analysis, and another for RNA extraction.

The remainder of the ceca was processed for histology as follows: The luminal contents were removed, washed with PBS, placed in tissue cassettes, and submerged in $10 \%$ formalin for 24 hours. The tissue cassettes were transferred to a $60 \%$ ethanol solution and then processed for paraffin embedding and staining with hematoxylin and eosin (H\&E).

Scoring was completed using the colitis index histological scoring system used by Berndt et $\mathrm{al}^{20}$ and adapted from Rachmilewitz et al. ${ }^{21}$ Briefly, the sections assessed on inflammation, transmural infiltration, cell wall thickening, and bleeding and scored on a scale ranging from 0 to 40 .

\section{RNA Extraction and Polymerase Chain Reaction (PCR) Array Analysis}

Total RNA from cecal tissue was isolated using TRIzol (Invitrogen, Carlsbad, CA) as directed by the manufacturer's protocol. The High-Capacity cDNA Reverse Transcription Kit (Applied Biosystems, Foster City, CA) was used to convert the total RNA to cDNA. Changes in host gene expression were measured using an array of gene-specific primers from Superarray (Frederick, MD), designed for the following targets ${ }^{22}$ : Jun, Cd80, Cd209a, Il12b, Irak3, Smad3, Arg1, Cd86, Mapk3, Il17a, Mapk8,
Tgfb1, Cd274, Creb1, Mapk1, Il1b, Tlr2, Tlr5, Vtcn1, Cx3cr1, Foxp3, Il2, Tlr4, Tlr9, Ccr2, Cxcl1, Tnfrsf18, Il23a, H2-DMb1, Tnf, Ccr7, Cxcl10, Infg, Il4, Nfkb1, Vegfa, Itgax, Cxc15, Il10, Il6, Mapk14, Gapdh, Cd40, Cxcl2, Il12a, Irak4, Pik3r1, ActB. The neutrophil marker, Ly-6G, was also quantified using the primer set from Sasmono et $\mathrm{al}^{23}$ (Ly-6G forward primer $5^{\prime}$-TGGACTCT CACAGAAGCAAAG- $3^{\prime}$ and reverse primer $5^{\prime}$-GCAGA GGTCTTCCTTCCAACA-3') as well as the Gapdh primer set from Cui et $\mathrm{al}^{24}$ (forward primer 5'-ACCACAGTC CATGCCATCAC-3' and reverse primer 5'-TCCAC CACCCTGTTGCTGTA-3). Quantization was performed using LightCycler 480 SYBR Green I Master and analyzed on a LightCycler 480 system (Roche Diagnostics, Indianapolis, IN) as directed by the manufacturer's protocol, with the following cycling conditions: $95^{\circ} \mathrm{C}$ activation for 10 minutes; 40 cycles of $95^{\circ} \mathrm{C}$ denaturation for 15 seconds, and $60^{\circ} \mathrm{C}$ annealing for 1 minute. Resulting threshold values were analyzed by calculating the $2^{-\Delta \Delta \mathrm{Ct}}$ values, using GAPDH as the reference, to find fold regulation compared to the no-DSS control. ${ }^{25-27}$

\section{DNA Extraction}

Genomic DNA was extracted from tissue using the Qiagen DNeasy Blood \& Tissue kit (Cat. No. 69504, Valencia, CA). Briefly, the tissue was incubated overnight in lysis buffer and proteinase $\mathrm{K}$ at $56^{\circ} \mathrm{C}$. The following day the enzyme was denatured at $95^{\circ} \mathrm{C}$ and DNA was purified using ethanol through filter columns, as directed by the manufacturer's protocol, and eluted in $30 \mu \mathrm{L}$ of elution buffer. This genomic DNA was then used in preparation of the clone libraries and T-RFLP.

\section{Clone Library Construction}

For clone library construction, the reaction was set up as described previously, ${ }^{28}$ with illustra PuReTaq Ready To Go PCR beads (GE Healthcare, Piscataway, NJ). Briefly, amplification by polymerase chain reaction was performed using broad-ranged primers, $\left(8 \mathrm{~F}, 5^{\prime}\right.$-AGAGTTT GATCCTGGCTCAG-3'; 1492R, 5'-GGTTACCTTGTTAC GACTT-3'). Amplicon purification was done using a commercial kit (GFX, GE Healthcare, Piscataway, NJ) as directed by the manufacturer. Products were ligated into the TOPO 4 vector (Invitrogen K4575-01) according to the manufacturer's specifications, and transformed into Escherichia coli. Colonies were picked into Luria Broth (LB) with carbenicillin $(50 \mu \mathrm{g} / \mathrm{mL})$ and grown overnight at $37^{\circ} \mathrm{C}$. Vector specific primers (M13F, 5'-CAGTCACGA CGTTGTAAAACGACGGC-3'; and M13R, 5'-CAGGA AACAGCTATGACCATG-3') were used to screen these colonies for bacterial clones containing the appropriate 1.5 $\mathrm{kb}$ amplicon insert. Partial $16 \mathrm{~S}$ sequences were determined by a single sequencing run using the $8 \mathrm{~F}$ primer at the 
Genomic Core at Michigan State University. Raw sequence data were processes through an automated "information pipeline" available through the Ribosomal Database Project (RDP) Website (http://rdp.cme.msu.edu/). Data were screened using the Chimera Check Program before uploading into the RDP. Following alignment of the sequences via myRDP ${ }^{29}$ distance matrices representing each of the libraries were downloaded and then taxonomic assignments designated (80\% confidence cutoff) using Classifier through the RDP website. These distance matrices were also input into mothur ${ }^{30}$ to be grouped into operational taxonomic units (OTUs). Analyses were done using a 97\% sequence similarity to denote species level. An input table was also generated for the EstimateS program via the RDP Pipeline. Estimate $\mathrm{S}^{31}$ was used to calculate ecological diversity indices from the aligned sequences. Dendrograms based on the Bray-Curtis similarity index were constructed using the Mega3 program. ${ }^{32}$

The 16S small subunit rRNA gene sequences obtained by clone library analysis were subjected to in silico T-RFLP analysis using the TRF-cut program in the ARB suite of programs. ${ }^{33}$ The $M s p I$ enzyme, a four base cutter, was used to calculate predicted terminal restriction fragments (TRFs) from the clone sequences. Histograms were then constructed displaying the relative abundance of these in silico generated TRFs and compared to the actual traces retrieved from the T-RFLP analysis described below. The fragment sizes obtained by T-RFLP analysis were compared to the sizes of the TRFs generated in silico from the cloned 16S sequences. These fragments were identified within two basepairs ( $\pm 2 \mathrm{bp}$ ) of the predicted TRFs. ${ }^{34}$

\section{T-RFLP Analysis}

T-RFLP was performed as described elsewhere ${ }^{35}$ using primers $1492 \mathrm{R}$ and FAM labeled 8F. In summary, genomic DNA was amplified using the aforementioned primers with PCR conditions: $94^{\circ} \mathrm{C}$ denaturation for 2 minutes; 30 cycles of denaturation at $94^{\circ} \mathrm{C}$ for 30 seconds; annealing at $58^{\circ} \mathrm{C}$ for 45 seconds; extension at $72^{\circ} \mathrm{C}$ for 90 seconds; and final extension at $72^{\circ} \mathrm{C}$ for 4 minutes. The $1.5-\mathrm{kb}$ PCR product was verified and purified with GFX columns (GFX, GE Healthcare), then subjected to digestion with the MspI restriction enzyme for 2 hours. The digested DNA was submitted for analysis to the Genome Technology Support Facility (GTSF) at Michigan State University. Traces were visualized using the program Genescan (Applied Biosystems).

T-RFLP Stats collection of programs (http://styx.ibest. uidaho.edu/ibest/research.html) ${ }^{36}$ were used to analyze the traces from the T-RFLP output data. Briefly, the peaks of each trace were binned into OTUs to produce data categorized by both OTUs and abundance. This was then used as input for the EstimateS (http://viceroyeebuconnedu/estimates $)^{31}$ program where the Bray-Curtis values were calcu- lated and used to compare the diversity among the communities. The Mega3 program $^{32}$ was used to construct dendrograms showing the relationship between community structures using the Bray-Curtis values. The significance differences between the community structures were calculated using the parsimony test from the mothur suite of programs.

\section{qPCR Analysis of Microbial Communities}

The quantity of $16 \mathrm{~S}$ rRNA operons in the samples relative to a single-copy host gene was measured using a primer/ probe set that targets a broad range of rRNA-encoding gene sequences $\left(5^{\prime}\right.$-TCCTACGGGAGGCAGCAGT- $\left.3^{\prime}\right)$, the reverse primer (5'-GGACTACCAGGGTATCTAATCCTGTT- $\left.3^{\prime}\right)$, and the probe (5'-[6-FAM]-203CGTATTACCGCGGCTG CTGGCAC-[TAMRA]- $\left.3^{\prime}\right) .{ }^{37}$ A primer/probe set targeting a 264-bp portion of the tumor necrosis factor alpha (TNF- $\alpha$ ) gene was used as a reference using 200 nanomoles of the forward (TNF $\alpha \_$mu_se; 5'-GGCTTTCCGAATTCACTG GAG- $3^{\prime}$ ) and reverse primers (TNF $\alpha \_m u \_a s ; 5^{\prime}$-CCCCGG CCTTCCAAATAAA $-3^{\prime}$ ), and 100 nanomoles of the probe (TNF $\alpha \_m u \_p r o b e ; 5 '$-[Cy5]-ATGTCCATTCCTGAGTTCTG CAAAGGGA-[Iowa Black RQ]- $\left.3^{\prime}\right){ }^{38}$ The reaction mix consists of LightCycler 480 Probes Master reaction mix (Roche) at $1 \times$ concentration, and appropriate primer/probe pair. Amplification of each gene was done under separate run conditions: cycling conditions for the $16 \mathrm{~S}$ target involved an activation step of $50^{\circ} \mathrm{C}$ for 2 minutes followed by $95^{\circ} \mathrm{C}$ for 10 minutes. Forty-five cycles was done at $95^{\circ} \mathrm{C}$ for 15 seconds and $60^{\circ} \mathrm{C}$ for 1 minute before holding. For the TNF reference gene cycling conditions included an activation step of $50^{\circ} \mathrm{C}$ for 2 minutes followed by $95^{\circ} \mathrm{C}$ for 10 minutes and 45 cycles of $95^{\circ} \mathrm{C}$ for 20 seconds and $64^{\circ} \mathrm{C}$ for 30 seconds. Calculations of $2^{-\Delta \Delta \mathrm{Ct}}$ were made to compare changes in the amount of $16 \mathrm{~S}$ from samples between treatment groups. ${ }^{21,22}$

\section{Statistical Analysis}

The nonparametric Kruskal-Wallis test was used to analyze histological scores. Differences in the abundances of specific operational taxonomic units among the treatment groups were analyzed by analysis of variance (ANOVA). Statistical differences in the dendrograms comparing distances between microbial communities were calculated using the parsimony test function in mothur. ${ }^{30}$ Probability values less than 0.05 were considered significantly different.

\section{RESULTS}

\section{DSS Treatment Leads to Inflammation of the Murine Intestinal Tract}

Wildtype mice were treated with 5\% DSS in drinking water and the gastrointestinal tissue examined histologically after 3 and 14 days of treatment. All animals survived the DSS treatment and no significant clinical signs were 

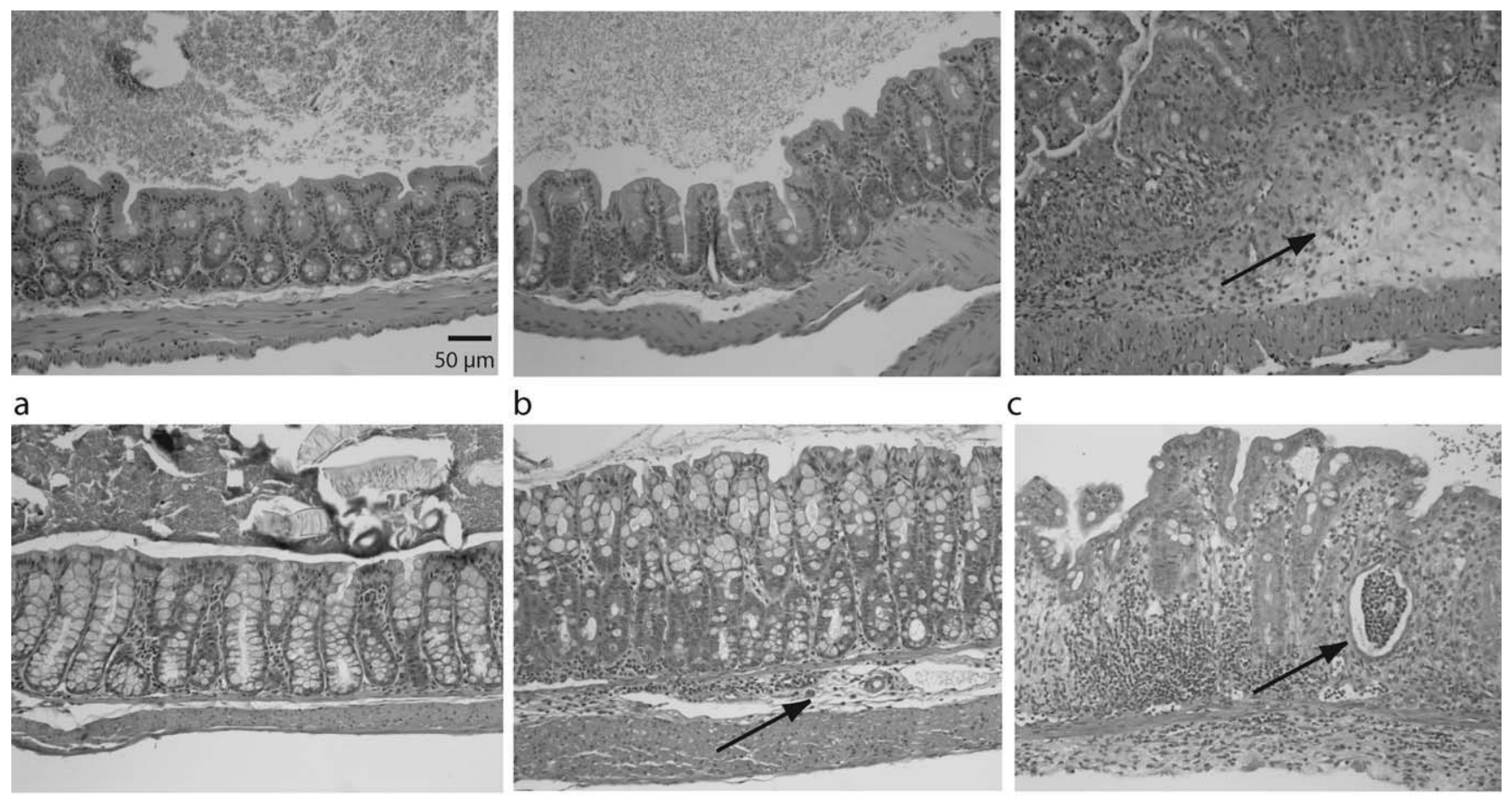

b

C
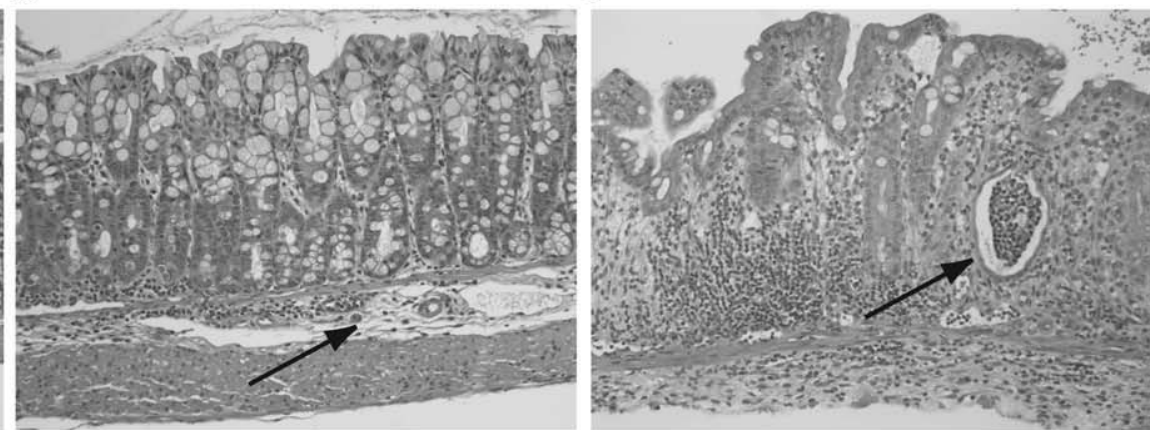

d

e

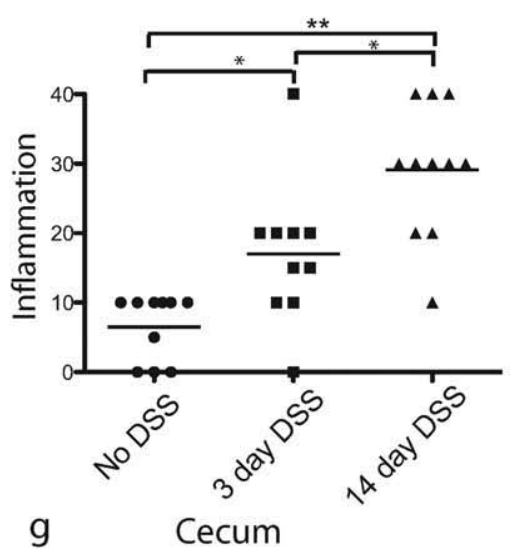

$f$

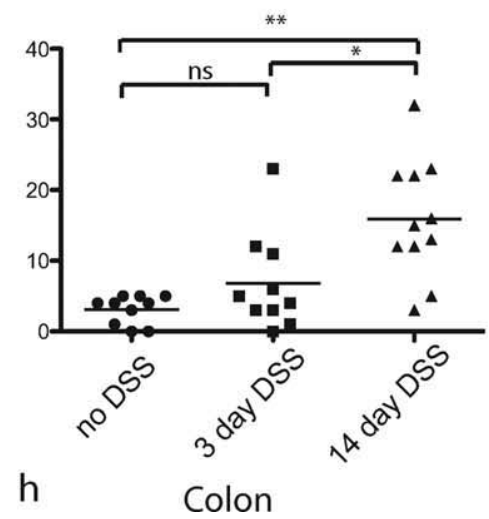

FIGURE 1. Histopathology in DSS-treated mice. H\&E-stained sections were prepared from the cecum of (a) untreated control mice (b) mice after 3 days of DSS treatment and (c) after 14 days of DSS. Arrow indicates submucosal edema. H\&E sections were also prepared from colon samples of (d) untreated controls (e) mice after 3 days of DSS treatment (arrow points to inflammation) and (f) animals after 14 days of DSS (arrow shows abscess). Histopathologic scores were calculated for sections from all 31 animals for $(\mathrm{g})$ cecum and (h) colon sections. Statistical analysis was done with the Kruskal-Wallis test. ${ }^{*} P<$ $0.05{ }^{* *} P<0.001$. Initial magnification $40 \times$.

noted. A mild inflammatory infiltrate developed in the cecum 3 days after DSS administration (Fig. 1b), with notable progression of disease after 14 days characterized the development of a massive inflammatory cell infiltrate and severe edema (Fig. 1c). These changes were significantly different from the control state $(P<0.05)$, as well as between the 3-day and 14-day treatment outcomes (Fig. 1g).

Similarly in the colon, a few animals developed morphological and inflammatory changes after 3 days of DSS treatment, but as a group these changes were not significant when compared to controls. However, following 14 days of DSS administration colonic tissue showed evidence of severe inflammation and edema, which was significantly greater than controls of animals after 3 days of treatment (Fig. 1h).

\section{Changes in Host Gene Expression Following DSS Treatment}

Using a quantitative PCR array, we found significant changes in the expression of ten host genes following DSS treatment (Fig. 2, Supp. Fig. 1). The expression of arginase 


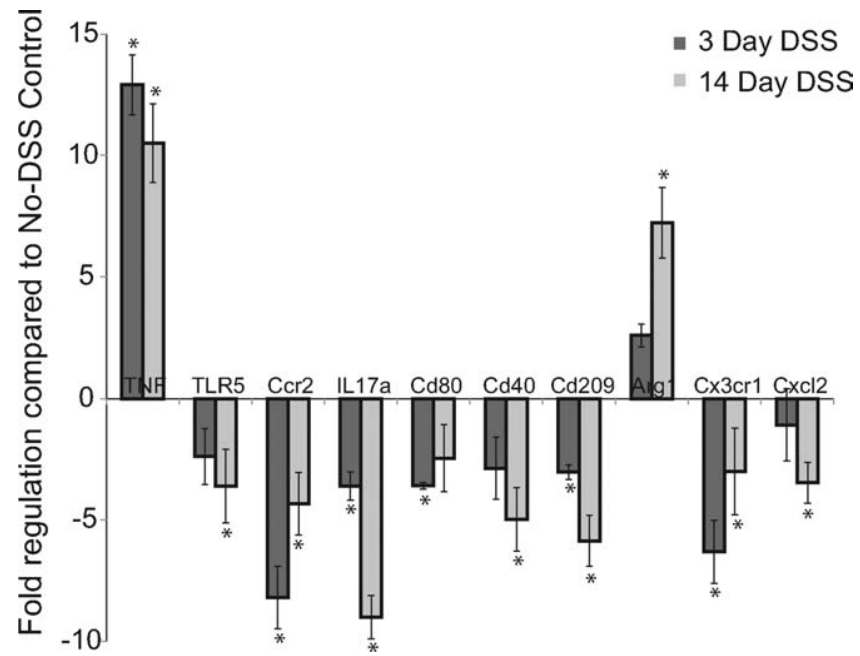

FIGURE 2. Changes in host gene expression after 3 days (dark bars) or 14 days (gray bars) \pm SD of DSS administration. Expression levels were compared to expression in tissue from animals not exposed to DSS. Statistical analysis was done using Student's $t$-test. Statistically significant differences $(P<0.05)$ are denoted by asterisks $\left(^{*}\right)$.

1 began to increase after 3 days and was significantly upregulated $\approx 7$-fold $(P<0.05)$ after 14 days of DSS administration. A significant increase in TNF- $\alpha$ was also seen after 3 days of DSS treatment, with a 12.91-fold upregulation $(P<0.05)$, and remained significantly upregulated at the 14-day timepoint.

The other eight genes were downregulated, compared to untreated controls. They were TLR 5, IL17, Ccr2, Cx3cr1, Cxcl2, Cd40, Cd80, and Cd209a. TLR 5 had significant downregulation after 14 days of DSS exposure, as did Cd40 and Cxcl2. Cd80 only showed a significant change after 3 days of DSS and only marginal change after 14 days. Ccr2, Cx3cr1, Cd209a, and IL17a showed significant downregulation both at the 3-day and 14-day timepoints.

The presence of neutrophils, as indicated by measuring the Ly-6G marker, increased significantly with DSS treatment with a $7.7 \pm 1.2$-fold increase after 3 days of DSS administration, and $14.0 \pm 1.8$-fold increase after 14 days of DSS (Supp. Fig. 1).

\section{Shifts in Gut Microbial Diversity Following DSS Treatment}

To obtain an overview of the status of the microbial community present in the gastrointestinal tract of each of the animals in this study, 16S T-RFLP analysis was performed on DNA extracted from cecal tissue. Analysis of the microbial communities in the cecum of all 31 animals in the study revealed that the structure of the microbial communities from DSS-treated mice were significantly different from the non-DSS controls $(P<0.001)$ (Supp. Fig. 2).
Since T-RFLP provides a broad overview of the community structure without information regarding changes in specific organisms, $16 \mathrm{~S}$ clone libraries were constructed to further investigate the microbial community in four representative mice from each treatment group. A total of 328 partial 16S rRNA-encoding gene sequences were retrieved from control animals (71, 84, 89, and 84 per animal), 364 sequences from animals 3 days after the initiation of DSS treatment $(88,92,93$, and 91), and 370 from the animals treated with 14 days of DSS (92, 94, 89, and 95). Clone library analysis confirmed the T-RFLP findings, indicating that the community compositions of the microbiota in DSS-treated animals were more similar to each other than they were to those of the control mice (Fig. 3, Supp. Fig. 4). The T-RFLP traces were compared to in silico TRFs of the clone libraries to verify that the OTUs matched the traces (Supp. Figs. 5-8). The communities in all DSStreated animals were significantly different from those in the non-DSS controls $(P<0.001$ by the parsimony test implemented in the analysis suite mothur ${ }^{30}$ ). Rarefaction analysis of the clone libraries revealed that DSS treatment resulted in a decrease in phylotype richness (Supp. Fig. 4). This finding was also supported by a decrease in the Simpson diversity index (1/D) following DSS treatment from $43.0 \pm 12.1$ to $21.3 \pm 8.3$ (3 days) and $19.7 \pm 7.0$ (14

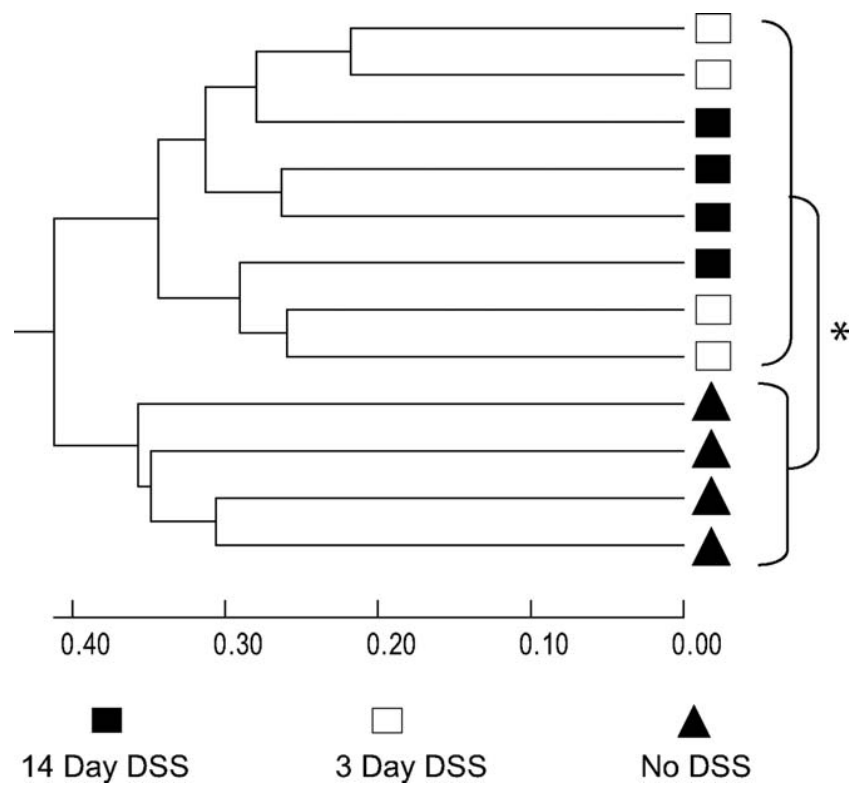

FIGURE 3. Comparison of the cecal community in control animals (black triangles) and in animals following 3 days of DSS treatment (white squares) and 14 days of treatment (black squares). Using an OTU definition of $97 \%$ similarity, the Bray-Curtis similarity metric was calculated for each pairwise comparison and then the results displayed in dendrogram format. Analysis by the parsimony test indicates that there is a statistically significant $\left({ }^{*} P<0.001\right)$ difference between the communities in control animals and in both groups of DSS-treated animals. 
A

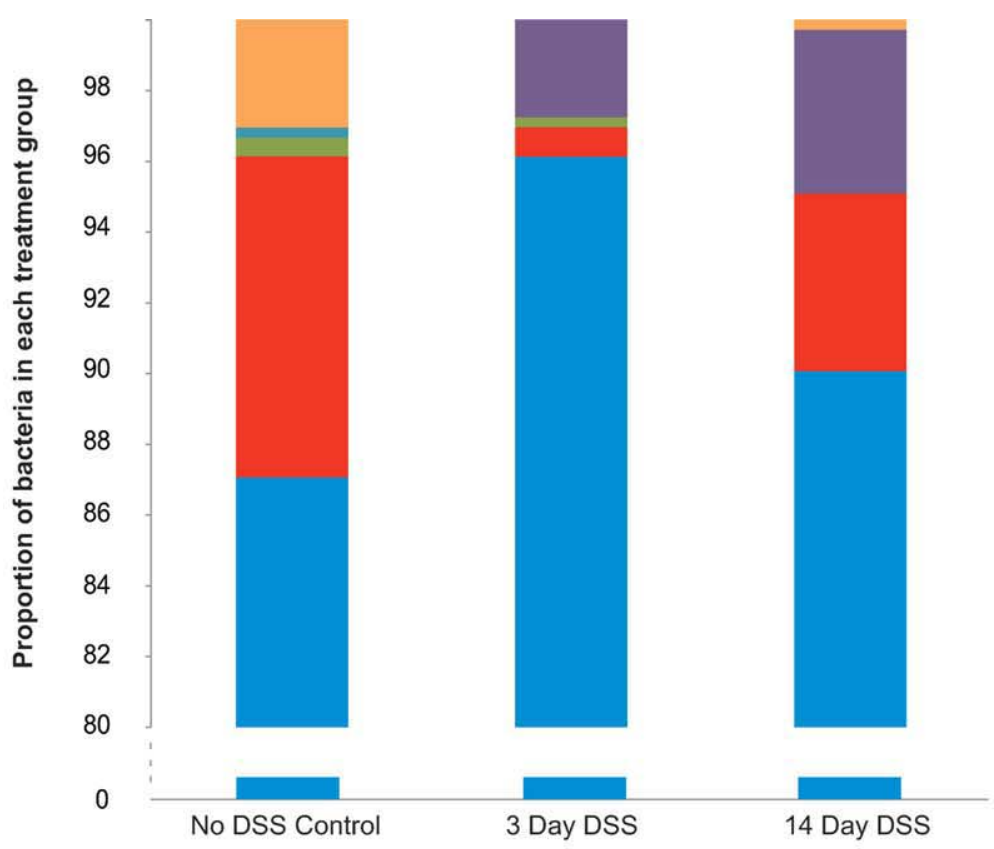

Phylum Level Assignment

mean proportion of bacteria per treatment group

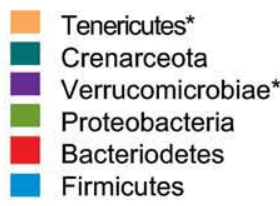

$3.14 \% \quad \pm 1.82$

$0.30 \% \quad \pm 0.60$

$0.00 \% \quad \pm 0.00$

$0.55 \% \pm 0.67$

$9.39 \% \quad \pm 5.00$

$86.90 \% \quad \pm 7.02$

B

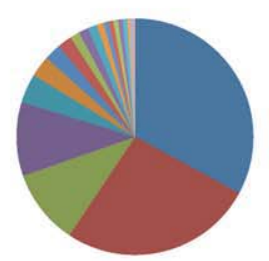

No DSS Control
$0.00 \% \pm 0.00$

$0.00 \% \pm 0.00$

$2.75 \% \pm 1.10$

$0.28 \% \pm 0.56$

$0.83 \% \pm 0.55$

$96.15 \% \pm 1.49$

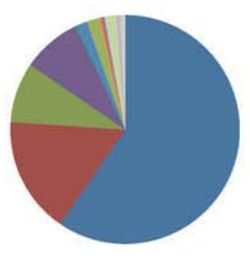

3 Day DSS
$0.27 \% \quad \pm 0.54$

$0.00 \% \quad \pm 0.00$

$4.64 \% \quad \pm 3.42$

$0.00 \% \quad \pm 0.00$

$5.04 \% \quad \pm 4.17$

$90.05 \% \quad \pm 3.31$

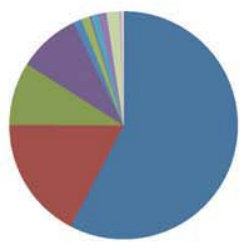

14 Day DSS

Assignments within Firmicutes mean proportion of bacteria per treatment group

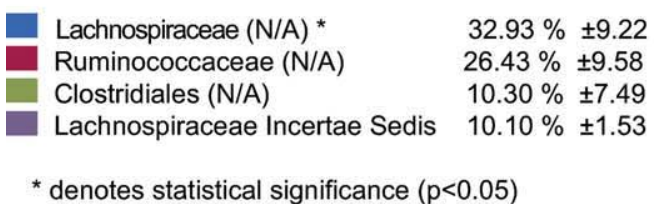

$57.69 \% \pm 11.64$

$17.39 \% \pm 5.74$

$8.95 \% \pm 4.03$

$8.75 \% \pm 3.46$

FIGURE 4. Changes in the cecal gut microbial community following DSS administration (A) at the phylum level (B) within the phylum Firmicutes. Analysis was done by ANOVA and statistical significance $(P<0.05)$ is denoted by asterisks $\left(^{*}\right)$.

days) $(P<0.05)$. However, quantitative PCR of the $16 \mathrm{~S}$ gene from each treatment group showed that there were no significant changes in overall bacterial biomass relative to the non-DSS control (fold change for 3 day DSS $=0.68 \pm$ $0.92 \mathrm{SD}, 14$ day DSS $=0.97 \pm 0.82 \mathrm{SD}$ ).

We analyzed changes in the relative abundance of specific phylotypes by classification of the 16S rRNA- encoding gene sequences. DSS treatment resulted in a decrease in members of the phyla Bacteriodetes and Tenericutes. Alterations in the microbial community were also apparent at lower taxonomic levels. For example, there was an increase in the unclassified genera under the family Lachnospiraceae (Fig. 4, Supp. Fig. 3). The majority of classified phylotypes in animals from all three experimental 
TABLE 1. Distribution of OTUs Across Treatments from Major Groups Within the Firmicutes

\begin{tabular}{lccc}
\hline & \multicolumn{3}{c}{ Clostridiales Ruminococcus Lachnospiracea } \\
& $N=17(\%)$ & $N=36(\%)$ & $N=65(\%)$ \\
\hline $\begin{array}{l}\text { Shared OTUs among } \\
\text { all groups }\end{array}$ & $6(35)$ & $16(44)$ & $15(23)$ \\
$\begin{array}{c}\text { OTUs in control } \\
\text { group only }\end{array}$ & $4(24)$ & $8(22)$ & $13(20)$ \\
$\begin{array}{l}\text { OTUs in 3 day DSS } \\
\text { group only }\end{array}$ & $2(12)$ & $4(11)$ & $8(12)$ \\
$\begin{array}{l}\text { OTUs in 14 day } \\
\text { DSS group only }\end{array}$ & $2(12)$ & $5(14)$ & $12(18)$ \\
$\begin{array}{c}\text { Shared OTU in } \\
\text { DSS-treated groups }\end{array}$ & $3(18)$ & $3(8)$ & $17(26)$ \\
\hline
\end{tabular}

groups were members of the phylum Firmicutes, which include the classes Clostridia, Mollicutes, and Bacilli. The Bacteriodetes was most abundant in the control animals and represented to a lesser extent in the DSS-treated groups (Fig. 4A, Supp. Fig. 3). A striking observation was that members of the phylum Verrucomicrobiae were detected only in the DSS-treated mice and not in the control mice (Fig. 4A).

Among members of the phylum Bacteriodetes the richness of the population did not change, indicating that the changes were mainly due to shift in abundances of different bacteria within this phylum. Among the Firmicutes, the unclassified genera among the families Ruminoccocaceae and Lachnospiraceae were most prevalent. These groups showed treatment related shifts as seen in the pie charts of Figure 4B. When analysis was done using 97\% sequence similarity, there was high richness among the Lachnospiraceae, compared to other major groups within the Firmicutes (Table 1), indicating increased diversity within this category $(P<0.05)$. These groups also showed treatment-related shifts (Table 1).

\section{DISCUSSION}

The indigenous gut microbiota are felt to play a key role in the pathogenesis of IBD. Much of the evidence for the involvement of intestinal bacteria in IBD comes from studies with murine models of disease. ${ }^{39,40}$ Many of these models have specific alterations in host defenses ranging from altered epithelial cell function to altered innate or adaptive immunity. ${ }^{41-44}$ These alterations in host defense result in abnormal interactions between the host and the indigenous microbiota that lead to disease. ${ }^{45}$ Evidence for this comes from the fact that antibiotic administration reduces the severity of disease in these models and rederivation of these mice to the germfree state prevents initiation of disease. ${ }^{13,46}$
Administration of DSS consistently triggers intestinal inflammation in rodents. Similar to other models, antibiotic administration has a beneficial effect in the system, again pointing to a role for the indigenous microbiota in the disease process. ${ }^{47}$ The effects of DSS on germfree mice have been variable. In some strains of mice more severe colitis is encountered, while early death, without the development of significant inflammation, is observed in others. ${ }^{48,49}$ Germfree IQI/Jic mice have a high mortality rate, compared to conventional mice, when given a concentration of 5\% DSS in drinking water. ${ }^{47}$ These mice died secondary to hemorrhage within 3 days after DSS treatment, indicating a protective function of the microbiota against the toxic effects of DSS on the gut epithelium. These results are consistent with the finding that antibiotic administration or genetic defects in TLR signaling also increased mortality in DSS-treated conventional mice. ${ }^{50,51}$ Differences in these two studies employing germfree mice likely reflects the differential effects of the two doses of DSS on the epithelium, but may also reflect differences in the composition of the microbiota of the conventional control animals. Since specific subsets of the "normal" microbiota have differing ability to initiate or sustain experimental colitis, ${ }^{47,52}$ these results emphasize the importance of characterizing the changes in the gut community associated with DSS-induced colitis across time.

Although it was originally suggested that IBD resulted from infection with an unknown bacterial pathogen, ${ }^{53}$ the more contemporary view is that entire shifts in the microbial community structure may be the trigger for disease. ${ }^{5}$ An altered gut microbial community has been associated with disease, as shown in human studies and animal models, ${ }^{54,55}$ suggesting that there is a change in abundance of bacteria that are necessary for maintaining homeostasis. ${ }^{56,57}$ After administering DSS to mice, we show that treatment results in changes in microbial community structure in the gut, also noted by others. ${ }^{51,58}$ Additionally, we found that these changes occur early (within 3 days) and are characterized by reduced overall diversity, while bacterial load remains unchanged. Some community members are below the limits of detection after DSS treatment, while others increase in abundance, demonstrating a selective effect of DSS treatment on specific members of the community. It has been shown that certain bacteria can depolymerize DSS and thus can grow in a DSS-rich environment better than others. ${ }^{59}$ This may explain the bloom of Verrucomicrobia that we observed in DSS-treated mice. This group of microbes, which has been found in mammalian intestine at very low abundances ${ }^{60}$ metabolize sulfur and degrade mucin. ${ }^{61}$ Therefore, perturbations of the community can cause previously underrepresented members of the community to become dominant, ${ }^{62}$ resulting in shifts in community structure, as seen in the phylum Bacteriodetes and families Ruminococcaceae and Lachnospiraceae. 
Another study has profiled the effects of DSS treatment on the microbial community. ${ }^{51}$ Similar to our study, it has been noted that shifts in the relative abundances of specific phylotypes were observed following DSS treatment, although the specific changes were different from what we observed. One obvious reason for these differences could be explained by the fact that different anatomic locations were sampled (cecal mucosa versus distal colonic contents), as well as length and concentrations of DSS administration. Additionally, it is likely that the baseline microbial communities were quite different between the animals in this study and the animals we used. Recent reports have demonstrated that the baseline microbiota can differ in genetically identical animals obtained from different sources and these differences can have a profound effect on host immune responses. ${ }^{63,64}$

Disease severity in the cecum increased after 3 days and 14 days post-DSS administration, concurrent with the changes observed in the microbial community, indicating a link between the changes in diversity of the microbiota and disease. Since shifts in the community structure can reduce beneficial members of the indigenous microbiota that act to maintain epithelial health, such as the production of short-chain fatty acids and stimulation of mucin production, ${ }^{57,65-68}$ it is likely that such changes can affect the host's inflammatory response. ${ }^{45,69}$

Others have suggested that DSS-induced colitis occurs due to breaches in the epithelial barrier with exposure to antigens produced by the luminal microbes. ${ }^{5,70-76}$ However, there is evidence that the detection of an altered microbial community is an important factor in the host response. ${ }^{77,78}$ Our data indicate that DSS induces changes in the microbiota, and together with a compromised barrier, appears to facilitate disease initiation and progression. It has been shown that the host responds to microbial-associated molecular patterns (MAMPS) via Toll-like receptors (TLRs) in the development of colitis. ${ }^{17,40,79} \mathrm{We}$ found a significant change in TLR 5, the expression of which was decreased as colitis progressed. It has been previously demonstrated that TLR 5 is downregulated in patients with severe ulcerative colitis, ${ }^{80}$ in concurrence with our data. However, the role of flagella in DSS colitis is not dependent on TLR5, ${ }^{40,81}$ suggesting that the downregulation is perhaps a response from the overstimulation from microbes through the deteriorating epithelial layer.

It is noteworthy that we saw an increase in arginase 1 after 14 days of DSS treatment. This could be due to the increase of neutrophils after DSS administration, as indicated by increased expression of the neutrophil marker Ly6-G. Arginase 1 also has a protective role in the Citrobacter rodentium model of colitis where enzyme inhibition aggravated disease. ${ }^{82}$ It has been seen that arginase expression increases with TNF- $\alpha$ /lipopolysaccharides (LPS) stimulation in human intestinal microvascular endothelial cells. ${ }^{83}$ With breaches in the epithelial layer that allows increased stimulation by LPS, together with an increase in TNF- $\alpha$ as seen here and in other studies with DSS, ${ }^{73,84}$ it follows that arginase expression is likely to be upregulated. Interestingly, although $\mathrm{TNF}-\alpha$ appeared to be downregulated at 14 days compared to 3 days, arginase was upregulated at 14 days compared to 3 days, consistent with the presumed role for this gene product in the epithelial repair response. To our knowledge, this study is the first to document the significant increase of this enzyme in DSS-induced colitis.

Although others have noted changes in the gut microbiota following DSS treatment ${ }^{51}$ after development of disease, this is the first study to monitor DSS-induced changes in the intestinal microbiota over time. In this study we not only corroborate that changes in the gut microbial community are associated with DSS-induced disease, but we also show that diversity of the microbiota changed as early as after 3 days and continued to 14 days of DSS administration. Early shifts in the microbial community were characterized with increases in abundances of microbes previously undetectable by the methods used. These altered communities are present at the onset of inflammation, and maintained as inflammation severity increased, reinforcing the idea that disease occurs, and persists, in the presence of an altered intestinal community. These early changes in the microbiota support the view that disturbance of the resident bacterial community structure may have a very important role in the onset of diseases such as IBD.

\section{ACKNOWLEDGMENTS}

We thank Jason Pratt for technical assistance and Dr. Courtney Robinson, Dr. Grace Chen, and Dr. Gary Huffnagle for helpful discussions and review of the article. We thank the Genomics Core at Michigan State University, where all the sequencing and T-RFLP data were generated, as well as the RDP staff for facilitating the work.

\section{REFERENCES}

1. Dethlefsen L, McFall-Ngai M, Relman DA. An ecological and evolutionary perspective on human-microbe mutualism and disease. Nature. 2007;449:811-818.

2. Ley RE, Peterson DA, Gordon JI. Ecological and evolutionary forces shaping microbial diversity in the human intestine. Cell. 2006;24: 837-848.

3. Round J, Mazmanian S. The gut microbiota shapes intestinal immune responses during health and disease. Nat Rev Immunol. 2009;9:313-323.

4. Subramanian S, Campbell BJ, Rhodes JM. Bacteria in the pathogenesis of inflammatory bowel disease. Curr Opin Infect Dis. 2006:475-484.

5. Packey C, Sartor R. Interplay of commensal and pathogenic bacteria, genetic mutations, and immunoregulatory defects in the pathogenesis of inflammatory bowel diseases. J Intern Med. 2008;263:597-606.

6. Swidsinski A, Ladhoff A, Pernthaler A, et al. Mucosal flora in inflammatory bowel disease. Gastroenterology. 2002;122:44-54.

7. Ogura Y, Bonen DK, Inohara N, et al. A frameshift mutation in NOD2 associated with susceptibility to Crohn's disease. Nature. 2001;411:603-606.

8. Kang SS, Bloom SM, Norian LA, et al. An antibiotic-responsive mouse model of fulminant ulcerative colitis. PLoS Med. 2008;5:e41. 
9. Swidsinski A, Weber J, Loening-Baucke V, et al. Spatial organization and composition of the mucosal flora in patients with inflammatory bowel disease. J Clin Microbiol. 2005;43:3380-3389.

10. Sellon RK, Tonkonogy S, Schultz M, et al. Resident enteric bacteria are necessary for development of spontaneous colitis and immune system activation in interleukin-10-deficient mice. Infect Immun. 1998; 66:5224-5231.

11. Madsen KL, Doyle JS, Tavernini MM, et al. Antibiotic therapy attenuates colitis in interleukin 10 gene-deficient mice. Gastroenterology. 2000;118:1094-1105.

12. Okayasu I, Hatakeyama S, Yamada M, et al. A novel method in the induction of reliable experimental acute and chronic ulcerative colitis in mice. Gastroenterology. 1990;98:694-702.

13. Rath H, Schultz M, Freitag R, et al. Different subsets of enteric bacteria induce and perpetuate experimental colitis in rats and mice. Infect Immun. 2001;69:2277-2285.

14. Tai EKK, Wu WKK, Wong HPS, et al. A new role for cathelicidin in ulcerative colitis in mice. Exp Biol Med (Maywood). 2007;232:799-808.

15. Kitajima S, Takuma S, Morimoto M. Changes in colonic mucosal permeability in mouse colitis induced with dextran sulfate sodium. Exp Anim. 1999;48:137-143.

16. Frank DN, St. Amand AL, Feldman RA, et al. Molecular-phylogenetic characterization of microbial community imbalances in human inflammatory bowel diseases. Proc Natl Acad Sci U S A. 2007;104: $13780-13785$.

17. Rakoff-Nahoum S, Paglino J, Eslami-Varzaneh F, et al. Recognition of commensal microflora by Toll-like receptors is required for intestinal homeostasis. Cell. 2004;118:229-241.

18. Vowinkel T, Kalogeris T, Mori M, et al. Impact of dextran sulfate sodium load on the severity of inflammation in experimental colitis. Dig Dis Sci. 2004;49:556-564.

19. Young VB, Knox KA, Pratt JS, et al. In vitro and in vivo characterization of Helicobacter hepaticus cytolethal distending toxin mutants. Infect Immun. 2004;72:2521-2527.

20. Berndt BE, Zhang M, Chen G-H, et al. The role of dendritic cells in the development of acute dextran sulfate sodium colitis. I Immunol. 2007; 179:6255-6262.

21. Rachmilewitz D, Karmeli F, Takabayashi K, et al. Immunostimulatory DNA ameliorates experimental and spontaneous murine colitis. Gastroenterology. 2002;122:1428-1441.

22. Lees CW, Zacharias WJ, Tremelling M, et al. Analysis of germline GLI1 variation implicates hedgehog signalling in the regulation of intestinal inflammatory pathways. PLoS Med. 2008;5:1761-1775.

23. Sasmono RT, Ehrnsperger A, Cronau SL, et al. Mouse neutrophilic granulocytes express mRNA encoding the macrophage colony-stimulating factor receptor (CSF-1R) as well as many other macrophagespecific transcripts and can transdifferentiate into macrophages in vitro in response to CSF-1. J Leukoc Biol. 2007;82:111-123.

24. Cui W, Cuartas E, Ke J, et al. CD200 and its receptors, CD200R, modulate bone mass via the differentiation of osteoclasts. PNAS. 2007; 104:14436-14441.

25. Pfaffl MW. A new mathematical model for relative quantification in real-time RT-PCR. Nucleic Acids Res. 2001;29:e45.

26. Guide to performing relative quantitation of gene expression using realtime quantitative PCR. Foster City, CA: Applied Biosystems; 2004.

27. Schmittgen TD, Livak KJ. Analyzing real-time PCR data by the comparative C(T) method. Nat Protoc. 2008;3:1101-1108.

28. Young VB, Schimdt TM. Antibiotic-associated diarrhea accompanied by large-scale alterations in the composition of the fecal microbiota. $J$ Clin Microbiol. 2004;42:1203-1206.

29. Cole JR, Chai B, Farris RJ, et al. The ribosomal database project (RDP-II): introducing myRDP space and quality controlled public data. Nuclic Acids Res. 2007;35:D169-172.

30. Schloss P, Westcott S, Ryabin T, et al. Introducing mothur: open source, platform-independent, community-supported software for describing and comparing microbial communities. Appl Environ Microbiol. 2009; 75:7537-7541.

31. Colwell RK. EstimateS: statistical estimation of species richness and shared species from samples. User's Guide and application. Available at: http://purloclcorg/estimates. 2006.
32. Kumar S, Tamura K, Nei M. MEGA3: integrated software for molecular evolutionary genetics analysis and sequence alignment. Brief Bioinform. 2004;5:150-163.

33. Ludwig W, Strunk O, Westram R, et al. ARB: a software environment for sequence data. Nucleic Acid Res. 2004;32:1363-1371.

34. Liu WT, Marsh TL, Cheng H, et al. Characterization of microbial diversity by determining terminal restriction fragment length polymorphisms of genes encoding 16S rRNA. Appl Environ Microbiol. 1997; 63:4516-4522.

35. Keuhl CJ, Wood HD, Marsh TL, et al. Colonization of the cecal mucosa by Helicobacter hepaticus impacts the diversity of the indigenous microbiota. Infect Immun. 2005;73:6952-6961.

36. Abdo Z, Schuette UME, Bent SJ, et al. Statistical methods for characterizing diversity of microbial communities by analysis of terminal restriction fragment length polymorphisms of $16 \mathrm{~S}$ rRNA genes. Environ Microbiol. 2006;8:929-938.

37. Martin FE, Nadkarni MA, Jacques NA, et al. Quantitative microbiological study of human carious dentine by culture and real-time PCR: association of anaerobes with histopathological changes in chronic pulpitis. J Clin Microbiol. 2002;40:1698-1704.

38. Nitsche A, Becker M, Junghahn I, et al. Quantification of human cells in NOD/SCID mice by duplex real-time polymerase-chain reaction. Haematologica. 2001;86:693-699.

39. Wirtz S, Neurath MF. Mouse models of inflammatory bowel disease. Adv Drug Deliv Rev. 2007;59:1073-1083.

40. Erridge C, Duncan S, Bereswill S, et al. The induction of colitis and ileitis in mice is associated with marked increases in intestinal concentrations of stimulants of TLRs 2, 4, and 5. PLoS. 2010;5:e9125.

41. Tang Y, Clayburgh DR, Mittal N, et al. Epithelial NF-kappaB enhances transmucosal fluid movement by altering tight junction protein composition after T cell activation. Am J Pathol 2010;176:158-167.

42. Shea-Donohue T, Thomas K, Cody MJ, et al. Mice deficient in the CXCR2 ligand, CXCL1 (KC/GRO-alpha), exhibit increased susceptibility to dextran sodium sulfate (DSS)-induced colitis. Innate Immun. 2008:14:117-124.

43. Blumberg RS, Saubermann LJ, Strober W. Animal models of mucosal inflammation and their relation to human inflammatory bowel disease. Curr Opin Immunol. 1999;11:648-656.

44. Belardelli F, Ferrantini M. Cytokines as a link between innate and adaptive antitumor immunity. Trends Immunol. 2002;23:201-208.

45. Abt MC, Artis D. The intestinal microbiota in health and disease: the influence of microbial products on immune cell homeostasis. Curr Opin Gastroenterol. 2009;25:496-502.

46. Sartor RB. Microbial influences in inflammatory bowel diseases. Gastroenterology. 2008;134:577-594.

47. Rath HC, Schultz M, Freitag R, et al. Different subsets of enteric bacteria induce and perpetuate experimental colitis in rats and mice. Infect Immun. 2001;69:2277-2285.

48. Hudcovic T, Stepankova R, Cebra J, et al. The role of microflora in the development of intestinal inflammation: acute and chronic colitis induced by dextran sulfate in germ-free and conventionally reared immunocompetent and immunodeficient mice. Folia Microbiol (Praha). 2001;46:565-572.

49. Kitajima S, Morimoto M, Sagara E, et al. Dextran sodium sulfateinduced colitis in germ-free IQI/Jic mice. Exp Anim. 2001;50:387-395.

50. Rakoff-Nahoum S, Paglino J, Eslami-Varzaneh F, et al. Recognition of commensal microflora by Toll-like receptors is required for intestinal homeostasis. Cell. 2004;118:229-241.

51. Heimesaat M, Fischer A, Siegmund B, et al. Shift towards pro-inflammatory intestinal bacteria aggravates acute murine colitis via Toll-like receptors 2 and 4. PLoS One. 2007;25:e662.

52. Hoentjen F, Harmsen HJ, Braat H, et al. Antibiotics with a selective aerobic or anaerobic spectrum have different therapeutic activities in various regions of the colon in interleukin 10 gene deficient mice. Gut. 2003;52:1721-1727.

53. Hansen R, Thomson JM, El-Omar EM, et al. The role of infection in the aetiology of inflammatory bowel disease. $J$ Gastroenterol. 2010;45:266-276.

54. Alexander S, Vera L-B, Mario V, et al. Active Crohn's disease and ulcerative colitis can be specifically diagnosed and monitored based on the biostructure of the fecal flora. Inflamm Bowel Dis. 2008;14:147-161. 
55. Suchodolski JS, Xenoulis P, Paddock CG, et al. Molecular analysis of the bacterial microbiota in duodenal biopsies from dogs with idiopathic inflammatory bowel disease. Vet Microbiol. 2010;142:394-400.

56. Maukonen J, Satokari R, Matto J, et al. Prevalence and temporal stability of selected clostridial groups in irritable bowel syndrome in relation to predominant faecal bacteria. J Med Microbiol. 2006;55: 625-633.

57. Cummings JH, Macfarlane GT. Role of intestinal bacteria in nutrient metabolism. JPEN J Parenter Enteral Nutr. 1997;21:357-365.

58. Deplancke B, Finster K, Graham WV, et al. Gastrointestinal and microbial responses to sulphate-supplemented drinking water in mice. Exp Biol Med. 2003;228:424-433.

59. Araki Y, Mukaisho K, Sugihara H, et al. Proteus mirabilis sp. intestinal microflora grow in a dextran sulfate sodium-rich environment. Int J Mol Med. 2010;25:203-208.

60. Wang M, Ahrne S, Jeppsson B, et al. Comparison of bacterial diversity along the human intestinal tract by direct cloning and sequencing of 16S rRNA genes. FEMS Microbiol Ecol. 2005:219-231.

61. Derrien M, Vaughan EE, Plugge CM, et al. Akkermansia muciniphila gen. nov., sp. nov., a human intestinal mucin-degrading bacterium. Int J Syst Evol Microbiol. 2004;54:1469-1476.

62. Antonopoulos D, Huse S, Morrison H, et al. Reproducible community dynamics of the gastrointestinal microbiota following antibiotic perturbation. Infect Immun. 2009;77:2367-2375.

63. Ivanov II, Frutos RL, Manel N, et al. Specific microbiota direct the differentiation of IL-17-producing T-helper cells in the mucosa of the small intestine. Cell. 2008;4:337-349.

64. Ivanov II, Atarashi K, Manel N, et al. Induction of intestinal Th17 cells by segmented filamentous bacteria. Cell. 2009;139:485-498.

65. Ahmad MS, Krishnan S, Ramakrishna BS, et al. Butyrate and glucose metabolism by colonocytes in experimental colitis in mice. Gut. 2000; 46:493-499.

66. Barcenilla A, Pryde SE, Martin JC, et al. Phylogenetic relationships of butyrate-producing bacteria from the human gut. Appl Environ Microbiol. 2000;66:1654-1661.

67. Arijs I, De Hertogh G, Lemaire K, et al. Mucosal gene expression of antimicrobial peptides in inflammatory bowel disease before and after first infliximab treatment. PLoS One. 2009;24:e7984.

68. Wostmann B. The germfree animal in nutritional studies. Аnnu Rev Nutr. 1981; 1.

69. Clarke TB, Davis KM, Lysenko ES, et al. Recognition of peptidoglycan from the microbiota by Nod1 enhances systemic innate immunity. Nat Med. 2010;16:160-161.
70. Johansson MEV, Phillipson M, Petersson J, et al. The inner of the two Muc2 mucin-dependent mucus layers in colon is devoid of bacteria. Proc Natl Acad Sci U S A. 2008;105:15064-15069.

71. Joerg DS, Svenja P, Maren A, et al. Epithelial tight junctions in intestinal inflammation. Ann N Y Acad Sci. 2009;1165:294-300.

72. Nenci A, Becker C, Wullaert A, et al. Epithelial NEMO links innate immunity to chronic intestinal inflammation. Nature. 2007; 446:557.

73. te Velde A, de Kort F, Sterrenburg E, et al. Comparative analysis of colonic gene expression of three experimental colitis models mimicking inflammatory bowel disease. Inflamm Bowel Dis. 2007;13: 325-330.

74. Podolsky D, Gerken G, Eyking A, et al. Colitis-associated variant of TLR2 causes impaired mucosal repair because of TFF3 deficiency. Gastroenterology. 2009;137:209-220.

75. Yan Y, Kolachala V, Dalmasso G, et al. Temporal and spatial analysis of clinical and molecular parameters in dextran sodium sulfate induced colitis. PLoS One. 2009;4:e6073.

76. Ito R, Kita M, Shin-Ya M, et al. Involvement of IL-17A in the pathogenesis of DSS-induced colitis in mice. Biochem Biophys Res Commun. 2008;377:12-16.

77. Stefan L, Dick SD, Eva J, et al. The role of the Lps gene in experimental ulcerative colitis in mice. APMIS. 1996;104:823-833.

78. Salzman NH, Bevins CL. Negative interactions with the microbiota: IBD. Adv Exp Med Biol. 2008;635:67-78.

79. Rakoff-Nahoum S, Hao L, Medzhitov R. Role of Toll-like receptors in spontaneous commensal-dependent colitis. Immunity. 2006;25: 319-329.

80. Stanislawowski M, Wierzbicki PM, Golab A, et al. Decreased Tolllike receptor-5 (TLR-5) expression in the mucosa of ulcerative colitis patients. J Physiol Pharmacol. 2009;60:71-75.

81. Sabine MI, Megan EH, Gijs H, et al. TLR5 is not required for flagellin-mediated exacerbation of DSS colitis. Inflamm Bowel Dis. 2010; 16:401-409.

82. Gobert AP, Cheng Y, Akhtar M, et al. Protective role of arginase in a mouse model of colitis. J Immunol. 2004;173:2109-2117.

83. Horowitz S, Binion DG, Nelson VM, et al. Increased arginase activity and endothelial dysfunction in human inflammatory bowel disease. Am J Physiol Gastroint Liver Physiol. 2007;292:G1323-1336.

84. Philip A, Nicholas CZ, Thuan N, et al. Distinct cytokine patterns identified from multiplex profiles of murine DSS and TNBS-induced colitis. Inflamm Bowel Dis. 2009;15:341-352. 